\title{
Performance Analysis for Distributed-Antenna OFDM Systems with Multiple CFOs Using Zero-Forcing Detection
}

\author{
Kai Deng ${ }^{1}$ and Wanzhi $\mathrm{Ma}^{2}$ \\ ${ }^{1}$ School of Physics and Electronic Engineering, Yibin University, Yibin 644000, \\ China, ${ }^{2}$ National Key Laboratory of Science and Technology on Communications, \\ University of Electronic Science and Technology of China, Chengdu 611731, \\ China \\ k_deng@126.com,mawanzhi@uestc.edu.cn
}

\begin{abstract}
This paper theoretically analyzes the performance of distributed-antenna orthogonal frequency division multiplexing (OFDM) systems with multiple carrier frequency offsets (CFO) using zero-forcing (ZF) detection. Considering both the large-scale fading and the multipath fading of the wireless channel, we derive the average power of the inter-carrier interference (ICI) due to the CFOs and thus obtain the expression for the signal to interference and noise ratio (SINR). By analyzing the underlying characteristics of the ZF detection, we address the reason for the inaccuracy of the conventional SINR expression and propose a novel modified SINR expression with better precision, and hence obtain a closed-form expression for the bit error rate (BER) performance with the presence of multiple CFOs. It is shown by the analytical and simulation results that the analytical SINR obtained by the conventional expression appears lower than actually while the analytical SINR and BER obtained by the expressions presented in this paper are more accurate and closer to the corresponding simulation results.
\end{abstract}

Keywords: Distributed antennas, OFDM, Carrier frequency offset (CFO), Inter-carrier interference (ICI), Zero-forcing (ZF) detection, Performance analysis

\section{Introduction}

With the rapid increase in the number of wireless users and the requirement for the amount of wireless services during the last five years, ultra dense cells and distributed multiple antennas have become the main features of the basic network architecture for the 4th and 5th generation (4G and 5G) mobile communications [1]-[2]. When deploying a distributed high density network, a large number of remote radio heads (RRH) are distributed at different geographic locations and connected to a pool of centralized baseband units (BBU) via optical fibers, forming a so-called cloud radio access network (C-RAN) architecture [3]-[4]. At the meantime, the technique of orthogonal frequency division multiplexing (OFDM) [5]-[6] has become the basic radio transmission technology of the physical layer for the $4 \mathrm{G}$ and $5 \mathrm{G}$ mobile communications, for it can convert the multipath wireless channel into parallel flat-fading subchannels and hence a simple single-tap frequency-domain equalizer can be adopted to equalize the channel easily.

In wireless communications, since different local oscillators are used at the base station (BS) and the mobile station (MS) respectively and the MS may move relatively to the BS as well [7]-[8], the carrier frequency offset (CFO) will unavoidably exist in the received signal. In particular, OFDM systems are very sensitive to the CFO, which damages the orthogonality among subcarriers, and causes inter-carrier interference (ICI) and hence degrades the performance [8]. Furthermore, as the RRHs are distributed at different geographic locations in distributed-antenna OFDM systems, either different local 
oscillators may be deployed for each RRH [9], or the transmitted signals from different RRHs may arrive at the MS from different angles [10], which results in multiple CFOs in the received signal. Therefore, it is very important to theoretically analyze the effects of the CFOs on the performance of the receiver, in order to provide reference to the link design and budget in practical engineering. On the other hand, some CFO estimation and compensation methods are usually adopted to estimate and compensate for the CFOs prior to detection (see, e.g., [8]-[14]). Hence performance analysis with the presence of CFOs is also required to evaluate the effectiveness of these CFO estimation or compensation methods.

The conventional theoretical analysis of the effects of the CFO on the performance of OFDM systems with a single antenna can be found in [7], which can be applied in distributed antenna OFDM systems with some straightforward modifications. Unfortunately, however, the analytical results appear inaccurate for distributed-antenna OFDM systems, and there is little research interest in performance analysis with better precision in the literature.

This paper theoretically analyzes the performance of distributed-antenna OFDM systems with multiple CFOs using zero-forcing (ZF) detection. Considering both the large-scale fading and the multipath fading of the wireless channel, we derive the average power of the ICI due to the CFOs and thus obtain the expression for the signal to interference and noise ratio (SINR). By analyzing the underlying characteristics of the ZF detection, we address the reason for the inaccuracy of the conventional SINR expression and propose a novel modified SINR expression with better precision, and hence obtain a closed-form expression for the bit error rate (BER) performance with the presence of multiple CFOs. The BER performance is also evaluated by computer simulations to validate the corresponding analytical results.

The subsequent parts of this paper are organized as follows. In Section 2, we propose the system model at hand. Section 3 addresses the performance analysis with the presence of multiple CFOs, where a novel modified SINR expression with better precision and thus a closed-form expression for the BER performance are derived. Some analytical and simulation results are presented in Section 4. And Section 5 is the conclusion of the paper.

\section{System Model}

We consider a $M_{\mathrm{T}} \times M_{\mathrm{R}}$ multiple-antenna OFDM system, where the $M_{\mathrm{T}}$ transmitting antennas are assumed to be located at different places while the $M_{\mathrm{R}}$ receiving antennas are assumed to be still located at the same place. This accounts for the downlink of a system with distributed antennas at the BS, where the CFOs between the BS and all the antennas at the MS are identical while the CFOs between the MS and each antenna at the BS may be different. We also assume that the channel and the CFOs remain almost constant during an OFDM symbol period [11].

Performing an $\mathrm{N}$-point inverse discrete Fourier transform (IDFT) on the data substreams that will be transmitted via the $M_{\mathrm{T}}$ transmitting antennas yields the time-domain signal vector of an OFDM symbol:

$$
\boldsymbol{x}(n)=\frac{1}{\sqrt{N}} \sum_{k=0}^{N-1} \boldsymbol{X}(k) e^{\mathrm{j} 2 \pi n k / N}, n=0,1, \cdots, N-1
$$

where $\boldsymbol{x}(n)=\left[x_{1}(n), x_{2}(n), \cdots, x_{M_{\mathrm{T}}}(n)\right]^{\mathrm{T}}$ is the time-domain signal vector of an OFDM symbol with $x_{i}(n)$ the time-domain signal of an OFDM symbol that will be transmitted via the $i$-th transmitting antenna, $X(k)=\left[X_{1}(k), X_{2}(k), \cdots, X_{M_{\mathrm{T}}}(k)\right]^{\mathrm{T}}$ is the data vector with $X_{i}(k)$ the data substreams that will be transmitted via the $i$-th transmitting antenna, and $N$ is the total number of subcarriers. 
At the receiver, performing an $N$-point discrete Fourier transform (DFT) on the received signal vector yields the demodulated frequency-domain signal vector with the presence of multiple CFOs [15]:

$$
\boldsymbol{Y}(k)=\underbrace{\boldsymbol{H}(k) \boldsymbol{S}(0) \boldsymbol{X}(k)}_{\text {Useful Signal }}+\underbrace{\sum_{l=0, l \neq k}^{N-1} \boldsymbol{H}(l) \boldsymbol{S}(l-k) \boldsymbol{X}(l)}_{\text {ICI }}+\boldsymbol{n}(k)
$$

where $\boldsymbol{Y}(k)=\left[Y_{1}(k), Y_{2}(k), \cdots, Y_{M_{\mathrm{R}}}(k)\right]^{\mathrm{T}}$ is the demodulated frequency-domain signal vector with $Y_{j}(k)$ the demodulated frequency-domain signal on the $j$-th receiving antenna. $\{\boldsymbol{H}(k)\}_{j i}=H_{i j}(k)$, where $H_{i j}(k)$ is the channel coefficient of the branch from the $i$-th transmitting antenna to the $j$-th receiving antenna at the $k$-th subcarrier, and $\boldsymbol{n}(k)$ is a Gaussian noise vector whose elements are independent identically distributed (i.i.d.) with zero-mean and variance $\sigma_{n}^{2}$.

At the right hand side of the equation in (2), the first term can be regarded as the useful signal and the second one can be regarded as the ICI due to the CFOs with $\boldsymbol{S}(k)=\operatorname{diag}\left\{S_{1}(k), \cdots, S_{M_{\mathrm{T}}}(k)\right\}$, whose diagonal elements are the ICI coefficients $\left\{S_{i}(k)\right\}$ and can be expressed by [15]

$$
S_{i}(k)=\frac{\sin \pi\left(k+\varepsilon_{i}\right)}{N \sin \frac{\pi}{N}\left(k+\varepsilon_{i}\right)} e^{\mathrm{j} \pi\left(1-\frac{1}{N}\right)\left(k+\varepsilon_{i}\right)}
$$

where $\varepsilon_{i}$ is the normalized $\mathrm{CFO}$ (ratio of the actual CFO to the subcarrier spacing) between the receiver and the $i$-th transmitting antenna, which can be regarded to be composed of the integral part, which is called integer frequency offset (IFO), and the fractional part, which is called fractional frequency offset (FFO). As the IFO only changes the order of the DFT outputs while the FFO actually yields the ICI [16], here we only involve the FFO in the CFO, i.e., it is assumed that $\left|\varepsilon_{i}\right| \leq 0.5, i=1,2, \cdots, M_{\mathrm{T}}$.

We consider both the large-scale fading (including mean path loss and log-normal shadow fading) [17] and the multipath fading of the wireless channel in the channel model, and assume that the multipath fading is Rayleigh fading. As a result, the channel coefficient $H_{i j}(k)$ can be written as $H_{i j}(k)=\sqrt{\xi_{i j}} h_{i j}(k)$, where $h_{i j}(k)$ is a zero-mean complex Gaussian random variable with unit variance, and $\xi_{i j}$ is a power gain factor that reflects the effects of the large-scale fading. As the transmitting antennas are located at different places while the receiving antennas are still located at the same place, $\left\{\xi_{i j}\right\}$ are possibly different for each transmitting antenna while the same for all the receiving antennas, thus the expression of $H_{i j}(k)$ can reduce to $H_{i j}(k)=\sqrt{\xi_{i}} h_{i j}(k)$. Additionally, it is also assumed that the channel coefficients for different branches from a transmitting antenna to a receiving antenna are mutually independent.

\section{Performance Analysis}

\subsection{Conventional SINR Expression}

We can see from (2) that the ICI on a given data substream comes not only from that data substream itself but also from other data substreams, and here we call the former intra-substream ICI and the latter inter-substream ICI. Without any loss in 
generality, in the following we will concentrate on the first data substream on the $j$ th receiving antenna and at the $k$-th subcarrier.

In most practical engineering cases, the total number of subcarriers $N$ is often large enough so that we can invoke the Central Limit Theorem, where the ICI can be modeled as a zero-mean white Gaussian noise [15]. Therefore, the SINR can be defined as the ratio of the average power of the useful signal for the first substream to the sum of the average ICI power (including intra-substream ICI and intersubstream ICI) and the noise power:

$$
\operatorname{SINR}=\frac{E\left[\left|H_{1, j}(k) X_{1}(k) S_{1}(0)\right|^{2}\right]}{E\left[\left|\sum_{i=1}^{M_{T}} \sum_{l=0, l \neq k}^{N-1} H_{i j}(l) X_{i}(l) S_{i}(l-k)\right|^{2}\right]+E\left[\left|n_{j}(k)\right|^{2}\right]} .
$$

Using the assumptions made to the channel, we have

$$
\operatorname{SINR}=\frac{\sigma_{s}^{2} \xi_{1}\left|S_{1}(0)\right|^{2}}{\sigma_{s}^{2} \sum_{i=1}^{M_{T}} \xi_{i} \sum_{l=0, l \neq k}^{N-1}\left|S_{i}(l-k)\right|^{2}+\sigma_{n}^{2}}=\frac{\rho \xi_{1}\left|S_{1}(0)\right|^{2}}{1+\rho \sum_{i=1}^{M_{T}} \xi_{i} \sum_{l=0, l \neq k}^{N-1}\left|S_{i}(l-k)\right|^{2}}
$$

where $\sigma_{s}^{2}=E\left[\left|X_{i}(k)\right|^{2}\right]$ is the average power of the data, and $\rho=\sigma_{s}^{2} / \sigma_{n}^{2}$ is the signal to noise power ratio (SNR).

When $N$ is sufficiently large, we have [7]

$$
\sum_{l=0, l \neq k}^{N-1}\left|S_{i}(l-k)\right|^{2}=1-\left|S_{i}(0)\right|^{2} .
$$

Hence

$$
\operatorname{SINR}=\frac{\rho \xi_{1}\left|S_{1}(0)\right|^{2}}{1+\rho \sum_{i=1}^{M_{T}} \xi_{i}\left(1-\left|S_{i}(0)\right|^{2}\right)} .
$$

Note that the above SINR expression is independent of the indices $j$ or $k$.

\subsection{Modified SINR Expression}

The conventional SINR expression in (7) appears inaccurate for distributedantenna OFDM systems using ZF detection. In the following we will address the reason for the inaccuracy and propose a novel modified SINR expression with better precision by analyzing the underlying characteristics of the ZF detection.

After demodulation by the DFT, a ZF detector [18] is applied to the demodulated frequency-domain signal vector to recover the original data substreams, i.e., the demodulated frequency-domain signal vector is multiplied by the pseudo-inverse of the channel coefficient matrix subcarrier by subcarrier:

$$
\boldsymbol{X}(k)=\boldsymbol{H}^{\dagger}(k) \boldsymbol{Y}(k)=\boldsymbol{S}(0) \boldsymbol{X}(k)+\sum_{l=0, l \neq k}^{N-1} \boldsymbol{H}^{\dagger}(k) \boldsymbol{H}(l) \boldsymbol{S}(l-k) \boldsymbol{X}(l)+\boldsymbol{n}^{\prime}(k)
$$

where $\boldsymbol{H}^{\dagger}(k)=\left(\boldsymbol{H}^{H}(k) \boldsymbol{H}(k)\right)^{-1} \boldsymbol{H}^{H}(k)$ denotes the pseudo-inverse of the channel coefficient matrix $\boldsymbol{H}(k), \quad \boldsymbol{X}(k)=\left[X_{1}(k), X_{2}(k), \cdots, X_{M_{\mathrm{T}}}(k)\right]^{\mathrm{T}}$ is the recovered data vector at the $k$-th subcarrier for decision with $X_{i}(k)$ the recovered data substream for the $i$-th transmitting antenna, and $\boldsymbol{n}^{\prime}(k)=\boldsymbol{H}^{\dagger}(k) \boldsymbol{n}(k)$ is the noise vector after detection. The second term at the right hand side of the equation in (8) can be regarded as the ICI after detection, also including intra-substream ICI and intersubstream ICI, which can be called post-detection intra-substream ICI and postdetection inter-substream ICI, respectively. The former is caused by the diagonal 
elements of the matrix $\boldsymbol{H}^{\dagger}(k) \boldsymbol{H}(l)$ and the latter is caused by the off-diagonal elements of the matrix $\boldsymbol{H}^{\dagger}(k) \boldsymbol{H}(l)$.

We can see from (3) that only the ICI coefficients introduced by the $q$ adjacent subcarriers are sufficiently large for a given subcarrier, where $q$ increases with the CFO, while the ICI introduced by the relatively distant subcarriers can be omitted, i.e.:

$$
\boldsymbol{S}(l-k) \approx \boldsymbol{0}_{M_{\mathrm{T}}}, \text { for }|l-k|>q
$$

where $\boldsymbol{0}_{M_{\mathrm{T}}}$ denotes an $M_{\mathrm{T}} \times M_{\mathrm{T}}$ matrix with all-zero elements. Hence (8) can be simplified as

$$
\boldsymbol{X}(k) \approx \boldsymbol{S}(0) \boldsymbol{X}(k)+\sum_{|l-k| \leq q} \boldsymbol{H}^{\dagger}(k) \boldsymbol{H}(l) \boldsymbol{S}(l-k) \boldsymbol{X}(l)+\boldsymbol{n}^{\prime}(k) .
$$

For wireless channels with relatively low delay spread, where the root-meansquare (r.m.s.) delay spread of the channel is sufficiently small so that the coherent bandwidth of the channel is significantly larger than the total bandwidth of the $2 q+1$ successive subcarriers, the frequency-domain response of the channel can be assumed to be approximately flat over $2 q+1$ successive subcarriers. Under this condition, we have

$$
\boldsymbol{H}(l) \approx \boldsymbol{H}(k),|l-k| \leq q
$$

and thus

$$
\boldsymbol{H}^{\dagger}(k) \boldsymbol{H}(l) \approx \boldsymbol{I}_{M_{\mathrm{T}}},|l-k| \leq q .
$$

where $\boldsymbol{I}_{M_{\mathrm{T}}}$ denotes an $M_{\mathrm{T}} \times M_{\mathrm{T}}$ unit matrix. Hence (10) can further reduce to

$$
\boldsymbol{X}(k) \approx \boldsymbol{S}(0) \boldsymbol{X}(k)+\sum_{|l-k| \leq q} \boldsymbol{S}(l-k) \boldsymbol{X}(l)+\boldsymbol{n}^{\prime}(k)
$$

or equivalently:

$$
X_{i}(k) \approx S_{i}(0) X_{i}(k)+\sum_{|l-k| \leq q} S_{i}(l-k) X_{i}(l)+n_{i}^{\prime}(k), i=1,2, \cdots, M_{\mathrm{T}}
$$

where $n_{i}^{\prime}(k)$ is the $i$-th element of $\boldsymbol{n}^{\prime}(k)$.

It can be observed from (14) that the inter-substream ICI has been eliminated by the ZF detection and the data substreams for different transmitting antennas have been separated and recovered after the ZF detection, only with their own intrasubstream ICI respectively whereas without any inter-substream ICI.

Based on the above analysis, it proves to be more reasonable to only consider the power of intra-substream ICI in the SINR expression and the power of intersubstream ICI should not be included. Therefore, the SINR expression in (7) should be modified as

$$
\operatorname{SINR}=\frac{\rho \xi_{1}\left|S_{1}(0)\right|^{2}}{1+\rho \xi_{1}\left(1-\left|S_{1}(0)\right|^{2}\right)} .
$$

\subsection{BER Analysis}

It is found in [19] that when the quadrature-phase-shift-keying (QPSK) modulation is employed the BER performance of multiple antenna systems with ZF detection over Rayleigh channels has a closed-form expression as

$$
\mathrm{BER}=J\left(M_{R}-M_{T}+1,1,2 \rho\right)
$$

with

$$
J(m, a, b)=\frac{1}{2}\left[1-\mu \sum_{k=0}^{m-1}\left(\begin{array}{c}
2 k \\
k
\end{array}\right)\left(\frac{1-\mu^{2}}{4}\right)^{k}\right]
$$


where $\mu=\sqrt{\frac{c}{1+c}}$ with $c=\frac{b}{2 a}$ and $\left(\begin{array}{c}2 k \\ k\end{array}\right)$ represents the binomial coefficient.

With the presence of multiple CFOs, since we have modeled the ICI as white Gaussian noise, by replacing the SNR in (16) with the SINR we have derived in (15) the BER performance of distributed-antenna OFDM systems with multiple CFOs using ZF detection can be obtained as

$$
\mathrm{BER}=J\left(M_{R}-M_{T}+1,1,2 \times \mathrm{SINR}\right) .
$$

\section{Analytical and Simulation Results}

This section presents some numerical results of the SINR and BER expressions we have derived above. The BER performance of the receiver with multiple CFOs is also evaluated by computer simulations to verify the corresponding analytical results.

A $2 \times 2$ distributed-antenna OFDM system is considered, i.e., $M_{\mathrm{T}}=M_{\mathrm{R}}=2$, with QPSK modulation and ZF detection, and the total number of subcarriers is $N=256$. The normalized CFOs are set as $\varepsilon_{1}=0.1$ and $\varepsilon_{2}=0.2$. The COST207 six-ray reduced typical urban (TU) channel model [17] is used, and the power gain factors are $\xi_{1}=1$ and $\xi_{2}=2$.

We first compare the numerical results of the conventional SINR expression (see (7)) and the modified SINR expression (see (15)) derived in this paper, as shown in Figure 1. It is observed that the analytical SINR obtained by the conventional expression is lower than that obtained by the modified expression. This is because the former involves both intra-substream ICI and inter-substream ICI, while the latter only involves the intra-substream ICI, which is closer to the actual case and more accurate according to the above analysis. Furthermore, the difference between the two analytical SINR results becomes larger as the SNR increases. This is because the impact of the ICI becomes dominant in the SINR expression at high SNR region and the difference between the two SINR expressions appears more significant.

To verify the analytical results, the BER performance of the receiver with multiple CFOs is also evaluated by computer simulations. Figure 2 compares the BER performance obtained by the conventional analysis, the proposed analysis and the simulations. It is shown that the analytical BER results derived in this paper are much closer to the corresponding simulation results. However, it is also worth noting the difference between the analytical BER and the simulated BER, the reason for which is summarized as follows.

(1) At low SNR region, the analytical BER is slightly lower than the simulated BER. This is because the r.m.s. delay spread of the COST207 TU channel is not sufficiently small and thus the frequency-domain response of the channel is not sufficiently flat over $2 q+1$ successive subcarriers so that the inter-substream ICI cannot be suppressed entirely by the ZF detection and hence the analytical SINR is slightly higher than actually.

(2) At high SNR region, the analytical BER is slightly higher than the simulated BER. This is due to the Gaussian approximation made to the ICI, which may introduce some inaccuracy when replacing the SNR in (16) with the SINR. This becomes more significant as the SNR increases for the impact of the ICI becomes dominant in the SINR. 


\section{Conclusion}

This paper theoretically analyzes the performance of distributed-antenna OFDM systems with multiple CFOs using ZF detection. By analyzing the underlying characteristics of the ZF detection, we have addressed the reason for the inaccuracy of the conventional SINR expression and proposed a novel modified SINR expression with better precision and hence obtained a closed-form expression for the BER performance with the presence of multiple CFOs. It is shown by the analytical and simulation results that the analytical SINR obtained by the conventional expression appears lower than actually while the analytical SINR and BER obtained by the expressions presented in this paper are more accurate and closer to the corresponding simulation results.

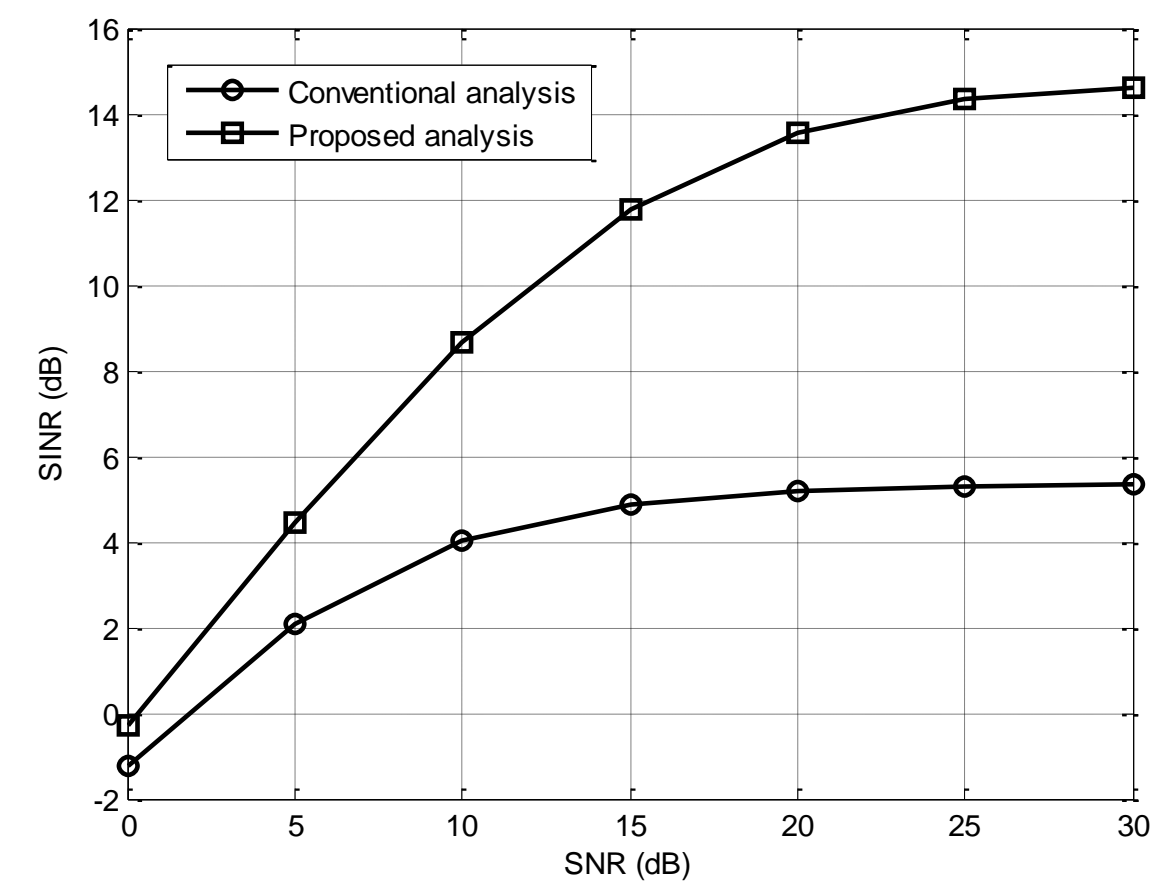

Figure 1. Comparison of Analytical Results for SINR. $M_{\mathrm{T}}=M_{\mathrm{R}}=2, N=256$, $\varepsilon_{1}=0.1, \varepsilon_{2}=0.2, \xi_{1}=1, \xi_{2}=2$, QPSK Modulation, and ZF Detection 


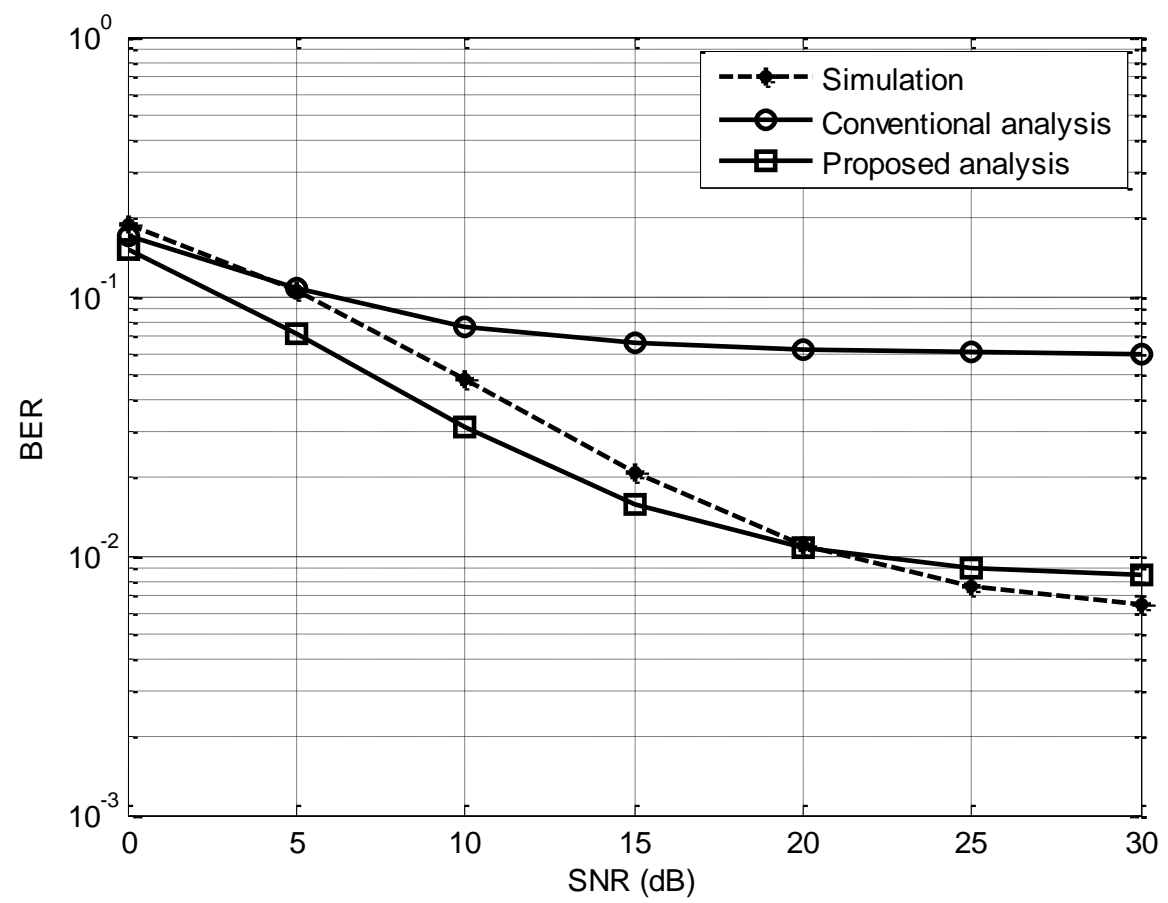

Figure 2. Comparison of Analytical and Simulation results for BER.

$M_{\mathrm{T}}=M_{\mathrm{R}}=2, N=256, \varepsilon_{1}=0.1, \varepsilon_{2}=0.2, \xi_{1}=1, \xi_{2}=2$, QPSK Modulation, and ZF

\section{Detection}

\section{Acknowledgements}

This research is financially supported in part by the National Natural Science Foundation of China under Grant No. 61201266 and the Scientific Innovation Foundation of Yibin City under Grant No. 2013ZSF008.

\section{References}

[1] S. Yunas, M. Valkama and J. Niemela, "Spectral and energy efficiency of ultra-dense networks under different deployment strategies", IEEE Commun. Mag, vol. 1, no. 53, (2015).

[2] S. Zhou, M. Zhao, X. Xu, J. Wang and Y. Yao, "Distributed wireless communication systems: a new architecture for future public wireless access", IEEE Commun. Mag, vol. 3, no. 41, (2003).

[3] J. Wu, Z. Zhang, Y. Hong and Y. Wen, "Cloud radio access network (C-RAN): a primer", IEEE Network, vol. 1, no. 29, (2015).

[4] R. Wang, H. Hu and X. Yang, "Potentials and challenges of C-RAN supporting multi-RATs toward 5G mobile networks", IEEE Access, vol. 2, (2014).

[5] J. A. C. Bingham, "Multicarrier modulation for data transmission: an idea whose time has come", IEEE Commun. Mag, vol. 5, no. 28, (1990).

[6] Z. Yi, G. Wang and H. Li, "Performance analysis of STC-OFDM system (in Chinese)", Journal of Harbin Univ. Sci. and Tech, vol. 5, no. 11, (2006).

[7] T. Pollet, M. V. Bladel and M. Moeneclaey, "BER sensitivity of OFDM systems to carrier frequency offset and Weiner phase noise", IEEE Trans. Commun, vol. 43, (1995).

[8] A. N. Mody and G. L. Stüber, "Synchronization for MIMO OFDM systems", Proceedings of the IEEE GLOBECOM, (2001).

[9] T. H. Pham, A. Nallanathan and Y. C. Liang, "Joint channel and frequency offset estimation in distributed MIMO flat-fading channels", IEEE Trans. Wireless Commun, vol. 2, no. 7, (2008).

[10] O. Besson and P. Stoica, "On parameter estimation of MIMO flat-fading channels with frequency offsets, "IEEE Trans. Signal Process, vol. 3, no. 51, (2003).

[11] S. Ahmed, S. Lambotharan, A. Jakobsson and J. A. Chambers, "MIMO frequency-selective channels with multiple frequency offsets: estimation and detection techniques", IEE Proc.-Commun, vol. 4, no. 152, (2005). 
[12] H. C. Wang and C. L. Wang, "A compact preamble design for synchronization in distributed MIMO OFDM systems", Proceedings of the IEEE VTC-Fall, (2011).

[13] J. Y. Lee, H. D. Lin and T. H. Sang, "An SFBC-OFDM receiver with MLSE equalizer to combat multiple frequency offsets", Proceedings of the IEEE ISCAS, (2012).

[14] K. Deng, "Frequency offset compensation for spatial multiplexing MIMO-OFDM systems with distributed transmitting antennas", Proceedings of the IEEE ICIST, (2012).

[15] D. N. Đao and C. Tellambura, "Inter-carrier interference self-cancellation space-frequency codes for MIMO-OFDM", IEEE Trans. Veh. Technol., vol. 5, no. 54, (2005).

[16] Y. H. Kim, I. Song, S. Yoon and S. R. Park, "An efficient frequency offset estimator for OFDM systems and its performance characteristics", IEEE Trans. Veh. Technol., vol. 5, no. 50, (2001).

[17] G. L. Stüber, "Principles of Mobile Communications", Kluwer, USA, (2002).

[18] A. J. Paulraj, R. U. Nabar and D. Gore, "Introduction to Space-Time Wireless Communications", Cambridge Univ. Press, UK, (2003).

[19] R. Xu and F. C. M. Lau, "Performance analysis for MIMO systems using zero forcing detector over fading channels", IEE Proc.-Commun., vol. 1, no. 153, (2006). 
International Journal of Future Generation Communication and Networking Vol. 9, No.7, (2016) 